\title{
Psychological and Exercise Intervention Training for Hemodialysis Patients: A Pilot Study
}

\author{
Michael MX Cai ${ }^{\mathrm{a}, \mathrm{b}}$, Sanjeev Beweja ${ }^{\mathrm{a}, \mathrm{b}}$, Rachel Reilly ${ }^{\mathrm{b}}$, Amy Clements ${ }^{\mathrm{a}}$, Annette Kent ${ }^{\mathrm{a}, \mathrm{b}}$, \\ Nicholas F Taylor ${ }^{\mathrm{c}}$, Robert J MacGinley, ${ }^{\mathrm{a}, \mathrm{d}}$, Stephen G Holt ${ }^{\mathrm{a}}$, Lawrence P McMahon ${ }^{\mathrm{a}}$
}

\begin{abstract}
Background: Patients with end stage kidney disease (ESKD) have a reduced health related quality of life (HRQoL). This pilot study examined the effect of a 6-month combined cognitive behavioral therapy (CBT) and muscle strength training program on hemodialysis patients.
\end{abstract}

Methods: The outcomes of interest included HRQoL as measured by Short-Form 36 version 2 (SF-36v2), cardiac depression scale, self-efficacy score, 6-minute walk distance (6MWD), physical activity and muscle strengths. Twenty-seven patients were recruited for intervention, but only eight completed the study.

Results: At baseline, participants who completed intervention (CI) had a higher role emotional score $(\mathrm{P}=0.028)$, role physical score $(\mathrm{P}=0.008)$ and self-efficacy score $(\mathrm{P}=0.019)$, compared to the participants who dropped out (ID). At the end of intervention, CI had improvements in physical functioning scores $(\mathrm{P}=0.040)$ and 6MWD $(\mathrm{P}=0.018)$, whereas ID had a decline in their role emotional scores $(\mathrm{P}=0.045)$.

Conclusions: The result of this study suggests that a combined CBT and physical training intervention can benefit hemodialysis patients, but non-adherence to therapy is common. Self-efficacy could play an important role in therapy adherence in the ESKD population.

Keywords: Quality of life; Depression; Renal failure; Hemodialysis; Cognitive behavioral therapy; Strength training; Self-efficacy

\footnotetext{
Manuscript accepted for publication April 15, 2014

${ }^{\mathrm{a}}$ Department of Renal Medicine, Eastern Health, Monash University, Melbourne, Australia

${ }^{b}$ Eastern Health Integrated Renal Service, Eastern Health, Melbourne, Australia

${ }^{\mathrm{c}}$ Department of Physiotherapy, La Trobe University, Melbourne, Australia

${ }^{\mathrm{d}}$ Corresponding author: Rob MacGinley, Department of Renal Medicine,

Level 2, 5 Arnold Street, Box Hill, Victoria 3128, Australia.

Email: rob.macginley@deakin.edu.au
}

doi: http://dx.doi.org/10.14740/wjnu156w

\section{Introduction}

Despite the development of transplantation and the availability of alternative dialysis modalities, in many countries thrice weekly in-center or satellite hemodialysis remains the predominant form of renal replacement therapy [1]. The long hours spent in a health-care setting, coupled with the medical and physical burden associated with end stage kidney disease (ESKD), often result in a reduced health related quality of life (HRQoL) [2].

It is therefore unsurprising that depression is common amongst patients with ESKD. While pharmaceutical intervention is sometimes used, it is not always appropriate or effective. Cognitive behavioral therapy (CBT) is well-established as an effective treatment for depression and anxiety in a range of populations [3], and has been shown to improve anxiety and depressive symptoms in hemodialysis patients [4-8].

In addition to psychological well-being, HRQoL is also associated with adequate physical functioning. Graded exercise programs have been shown to improve HRQoL in hemodialysis patients [9]. However, few if any, studies have assessed whether combined exercise and psychological intervention may improve HRQoL in hemodialysis patients.

The objective of the psychological and exercise intervention study (PEIDS) was to assess the effect of a combined exercise program and group psychological therapy on HRQoL, psychological well-being and physical activity on patients with ESKD.

\section{Materials and Methods}

\section{Study design}

PEIDS was a pilot prospective intervention control study. All patients receiving in-center, thrice-weekly hemodialysis with the Eastern Health Integrated Renal Service were screened for eligibility. Exclusion criteria included dialysis for less than 12 months, severe anemia (hemoglobin concentration $<105 \mathrm{~g} / \mathrm{L}$ for at least 3 months), uncontrolled second- 


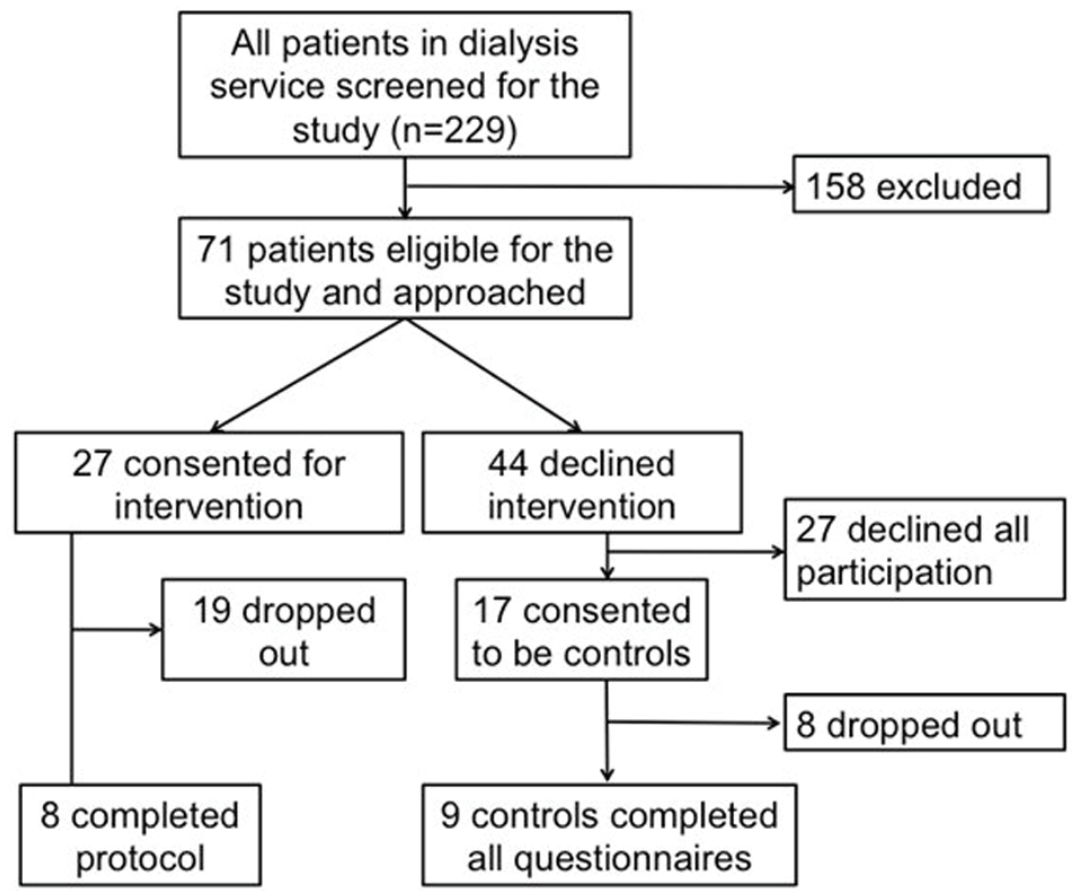

Figure 1. Flow diagram of patient recruitment and drop-out.

ary hyperparathyroidism ( $<40 \mathrm{pmol} / \mathrm{L}$ for at least 3 months), inadequate dialysis (urea reduction ratio of $<65 \%$ ), younger than 18 or older than 85 years, pregnancy, uncontrolled hypertension (consistent pre-dialysis BP $>160 / 100 \mathrm{~mm} \mathrm{Hg}$ ), unstable cardiovascular disease, unable to converse in English, or under active psychiatric or psychological care at the time of screening. After protocol approval by the Eastern Health Ethics Committee, all patients gave written, informed consent to participate in the study.

After screening, eligible patients were approached to participate in the intervention arm of the study. Those who agreed had 6 months of psychological intervention consisting of 13 psychologist-led, structured, CBT group sessions, each lasting an hour. The CBT intervention was adapted from a 13-session manual developed for cardiac patients by a senior clinical psychologist at the health service. The primary goal of the CBT intervention was to help patients understand and manage the emotional stress that can accompany chronic kidney disease. The exercise intervention was added to the second 3 months of the study. This was comprised of $12 \times$ weekly physiotherapist-led resistance exercise group sessions, each lasting $30-45 \mathrm{~min}$ in duration. All exercises were completed using graded resistance bands and body weight. Participants were also encouraged to follow an exercise program during dialysis twice weekly and to complete an exercise log book. Patients who declined intervention were invited to participate in the control arm. Data were collected at baseline, 3 months and at study end (6 months).

\section{Measures}

The primary outcome measure was change in HRQoL as measured by Short-Form 36 version 2 (SF-36v2), using Australian population norms [10]. Secondary outcome measures included the cardiac depression scale (CDS), a 26-item scale validated for use with cardiac patients [11], the exercise selfefficacy scale (EES) [12], 6-minute walk distance (6MWD) [13], muscle strength (measured using a hand-held dynamometer), and week-long physical activity levels measured with an accelerometer-based activity monitor ( ActivePAL ${ }^{\circledR}$ system) [14]. The 6WMD, muscle strength and physical activity levels were measured only in intervention patients. Participants were requested to have follow-up outcome assessments even if they dropped out of the intervention arm.

\section{Statistical analysis}

All baseline variables were assessed for normality and the appropriate summary statistics were used. Parametric data were expressed as means (standard deviation), and non-parametric data were expressed as medians (interquartile range). Due to the unexpectedly high number of dropouts from the intervention arm, baseline and serial analyses were formulated on the following three groups of participants: intervention completed (IC), began intervention but dropped out (ID), and controls (CO). The paired t-test was used to determine the difference in outcomes across the time points. All statistical analysis was performed using SPSS version 18, IBM Inc. 
Table 1. Baseline Demographic and Clinical Characteristics

\begin{tabular}{llllll}
\hline & IC (n=8) & ID (n= 19) & P value & CO (n= 17) & P value $^{2}$ \\
& & & & & \\
\hline Age (years) & $64(6)$ & $70(11)$ & 0.268 & $67(15)$ & 0.056 \\
Male gender & $7(88 \%)$ & $11(58 \%)$ & 0.149 & $9(53 \%)$ & 0.276 \\
Dialysis vintage (months) & $39(31)$ & $46(35)$ & 0.714 & $50(38)$ & 0.462 \\
Body mass index & $26(3)$ & $27(5)$ & 0.165 & $26(5)$ & 0.853 \\
Diabetes, n (\%) & $1(13 \%)$ & $7(37 \%)$ & 0.216 & $7(41 \%)$ & 0.321 \\
Hypertension, n (\%) & $6(75 \%)$ & $16(84 \%)$ & 0.472 & $14(82 \%)$ & 0.635 \\
Myocardial infarct, n (\%) & 0 & $3(16 \%)$ & 0.331 & $3(18 \%)$ & 0.426 \\
Peripheral vascular disease, $\mathrm{n}(\%)$ & 0 & $4(21 \%)$ & 0.221 & $2(12 \%)$ & 0.574 \\
Cerebral vascular disease, $\mathrm{n}(\%)$ & $1(13 \%)$ & $1(5 \%)$ & 0.513 & $3(18 \%)$ & 0.285 \\
History of anxiety, n (\%) & 0 & $1(5 \%)$ & 0.704 & 0 & 0.614 \\
History of depression, $\mathrm{n}(\%)$ & 0 & $2(11 \%)$ & 0.487 & $3(18 \%)$ & 0.285 \\
Systolic BP (mm Hg) & $137(15)$ & $139(14)$ & 0.447 & $143(16)$ & 0.348 \\
Diastolic BP (mm Hg) & $76(15)$ & $67(14)$ & 0.877 & $70(13)$ & 0.967 \\
Hb (g/L) & $114(10)$ & $120(10)$ & 0.856 & $115(10)$ & 0.251 \\
Urea reduction ratio & $0.74(0.04)$ & $0.75(0.03)$ & 0.424 & $0.76(0.05)$ & 0.956 \\
Serum albumin (g/L) & $37(4)$ & $36(3)$ & 0.433 & $35(4)$ & 0.528 \\
Total protein (g/L) & $66(4)$ & $67(5)$ & 0.273 & $66(5)$ & 0.651 \\
CRP (mg/L) & $8(3)$ & $19(7)$ & 0.074 & $5(4)$ & 0.587 \\
Phosphate (mmol/L) & $1.69(0.27)$ & $1.54(0.38)$ & 0.384 & $1.57(0.34)$ & 0.838 \\
PTH (pmol/L) & $27(12)$ & $44(26)$ & 0.053 & $39(29)$ & 0.984 \\
\hline
\end{tabular}

${ }^{1} \mathrm{IC}$ vs. CO; ${ }^{2}$ Intervention arm (IC and ID) vs. control arm (CO). Values (except nominal variables) are expressed in mean (SD).

A P value $<0.05$ indicated statistical significance.

\section{Results}

\section{Participants}

Of 229 patients screened for eligibility (Fig. 1), 71 (31\%) were found eligible. Most patients were excluded because they had not been dialyzing for the stipulated 12 months, could not converse in English or had a co-existing psychiatric illness requiring specialist care. Twenty-seven patients gave informed consent to participate in the intervention arm. Of the remaining 44 patients, 17 agreed to participate in the control arm. Nineteen (70\%) participants from the intervention arm dropped out of the study. Most dropouts occurred within the first 3 months. Their reasons included inability or unwillingness to meet the time commitment $(n=7)$, finding psychological intervention unhelpful $(n=4)$, physical illness requiring extended hospitalization ( $\mathrm{n}=2)$, group dissolution $(\mathrm{n}=2)$, post dialysis fatigue $(\mathrm{n}=1)$, transplantation $(\mathrm{n}=1)$, migration $(n=1)$ and death $(n=2)$.

\section{Baseline analysis}

The baseline demographic and clinical characteristics are shown in Table 1 . There were no statistically significant differences between the three groups. Only five (11\%) participants had a previous diagnosis of depression, one of whom had a co-existing anxiety disorder.

Baseline outcomes data are outlined in Table 2. On most SF-36 domains, participants scored lower than the general population means, namely $<50$. Compared to the IC group, the ID group had lower numerical scores across all domains. The difference between "bodily pain" and "role emotional" domains was statistically significant. There was also a trend towards a lower mental composite summary $(\mathrm{P}=0.06)$ for ID compared with IC. There were no significant differences 
Table 2. Baseline Outcomes of Interest

\begin{tabular}{|c|c|c|c|c|c|}
\hline & IC $(n=8)$ & ID $(n=19)$ & P value & $\operatorname{CO}(n=17)$ & $P$ value \\
\hline \multicolumn{6}{|l|}{ Health related quality of life } \\
\hline \multicolumn{6}{|l|}{ SF-36 } \\
\hline $\mathrm{PF}$ & $37(12)$ & $33(14)$ & 0.472 & $41(11)$ & 0.167 \\
\hline $\mathrm{RP}$ & $39(13)$ & $37(12)$ & 0.807 & $42(11)$ & 0.289 \\
\hline BP & $48(14)$ & $35(13)$ & 0.028 & $45(12)$ & 0.238 \\
\hline $\mathrm{GH}$ & $40(13)$ & $38(8)$ & 0.708 & $41(10)$ & 0.556 \\
\hline VT & $44(11)$ & $44(8)$ & 0.974 & $47(11)$ & 0.280 \\
\hline $\mathrm{SF}$ & $44(10)$ & $39(12)$ & 0.307 & $45(12)$ & 0.300 \\
\hline $\mathrm{RE}$ & $49(9)$ & $34(17)$ & 0.008 & $37(14)$ & 0.961 \\
\hline $\mathrm{MH}$ & $51(8)$ & $42(14)$ & 0.072 & $47(10)$ & 0.671 \\
\hline PCS & $38(16)$ & $36(12)$ & 0.747 & $42(9)$ & 0.139 \\
\hline MCS & $52(9)$ & $42(16)$ & 0.060 & $45(10)$ & 0.962 \\
\hline CDS & $79(32)$ & $93(30)$ & 0.299 & $81(20)$ & 0.371 \\
\hline EES & $121(42)$ & $82(28)$ & 0.019 & $97(38)$ & 0.788 \\
\hline \multicolumn{6}{|l|}{ Physical performance } \\
\hline 6MWD (m) & $429(75)$ & $334(94)$ & 0.018 & - & - \\
\hline$\%$ of predicted $6 \mathrm{MWD}^{*}$ & $78(12)$ & $74(25)$ & 0.684 & - & - \\
\hline Time spent recumbent $(\mathrm{h})$ & $19(3)$ & $21(2)$ & 0.156 & - & - \\
\hline Steps per day $(\times 1,000)$ & $6.8(3.5)$ & $3.7(2.4)$ & 0.063 & - & - \\
\hline \multicolumn{6}{|l|}{ Muscle strength $(\mathrm{kg})$} \\
\hline Chest press & $12(5)$ & $12(6)$ & 0.962 & - & - \\
\hline Knee extension & $17(5)$ & $16(5)$ & 0.571 & - & - \\
\hline Hip extension & $16(3)$ & $15(5)$ & 0.780 & - & - \\
\hline
\end{tabular}

PF: physical functioning; RP: role physical; BP: bodily pain; GH: general health; VT: vitality; SF: social functioning; RE: role emotional, PCS: physical composite summary; MCS: mental composite summary; CDS: cardiac depression scale; EES: exercise efficacy scale; *: for age-matched controls. All values are expressed in mean (SD).

in baseline SF-36 scores between intervention and control patients. Across the entire cohort, the mean CDS was 86, and $39 \%$ of all participants scored more than 95 , indicating a diagnosis of major depressive episode [15]. There was no significant difference in CDS scores between the three groups. Participants' perception of their own exercise efficacy at baseline was significantly lower in the ID group compared to the IC group, but there were no differences between the intervention group as a whole (IC and ID) and the $\mathrm{CO}$ group. ID participants had a significantly shorter 6MWD at baseline than IC participants. Based on the predicted 6MWD derived from the normal population, after adjustment for sex, age, weight and height, the intervention participants walked on average $76 \%$ of their predicted values [16]. There was no significant difference in the percentage of predicted values between IC and ID group.

Participants were markedly sedentary, spending on average of $20 \mathrm{~h}$ per day lying or sitting. The number of steps walked per day was well below the recommended 10,000. Steps taken were numerically higher in IC participants, but observed differences did not reach statistical significance. Muscle strength was markedly reduced in the study cohort when compared to an age and sex matched healthy cohort [17], on average $45 \%$ of expected in healthy subjects for 


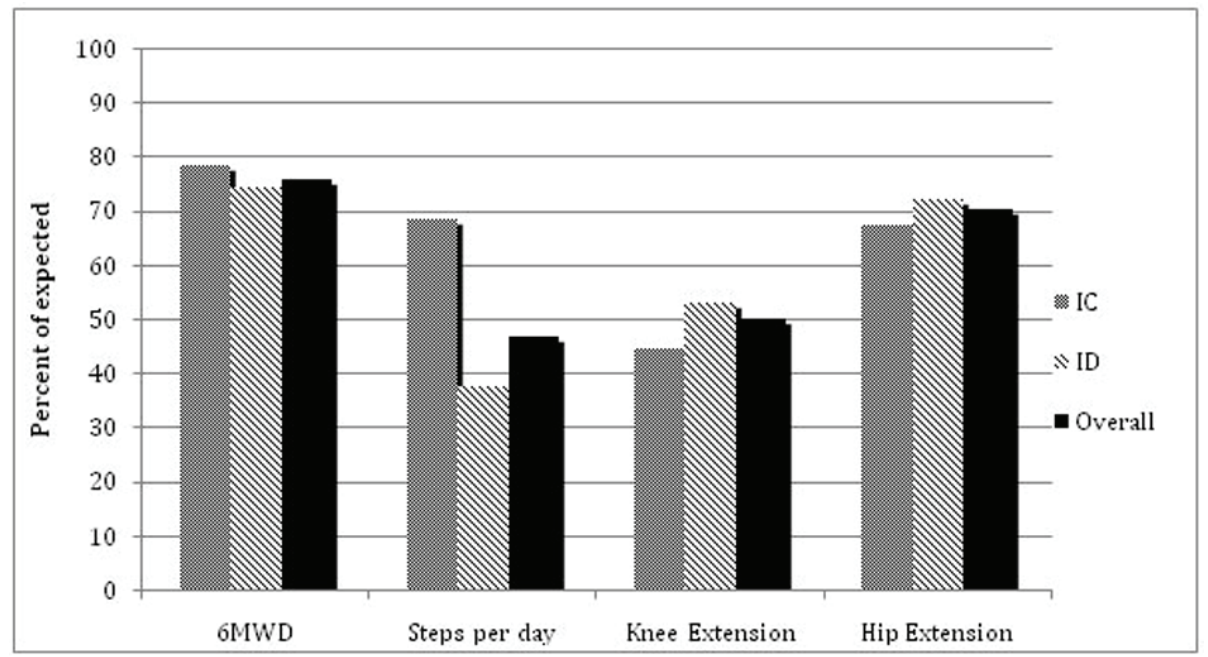

Figure 2. Comparison of selected baseline physical parameters between groups against normal expectations (6MWD were adjusted for age, sex, weight and height; muscle strengths were adjusted for age and sex; steps per day were compared against the recommended value of 10,000 steps per day). Values are expressed as a percentage of the expected value.

knee extension and $70 \%$ for hip extension, respectively. There was no difference in baseline muscle strength between the IC and ID group. Figure 2 illustrates the marked differences in the selected baseline physical parameters against the normal population.

Four participants in the ID group withdrew from the study due to physical illnesses. The baseline data for the ID and IC groups were accordingly re-analyzed with these four patients removed from the data set. The SF-36 role emotional (RE) domain remained significantly lower in the ID compared to the IC group ( $33 \pm 18$ vs. $49 \pm 9, \mathrm{P}=0.013)$. The EES also remained significantly lower in the ID group compared to the IC group (mean $86 \pm 25$ vs. $121 \pm 42, \mathrm{P}=0.031$ ).

\section{Serial analysis}

\section{Primary endpoints}

Figure 3 demonstrates the mean change in SF-36 between baseline and at 6 months. The IC group had a significantly improved physical functioning domain $(\mathrm{P}=0.040)$, whereas the ID group showed a decline in the RE domain $(\mathrm{P}=0.045)$.

\section{Secondary endpoints}

Changes in other endpoints of the study are illustrated in Table 3. Overall, there were no significant changes in CDS or EES across the three groups. Serial exercise measurements for the IC group showed a $23 \%$ improvement in the 6MWD between the start and end of the study, which was statistically significant. No statistical difference was observed in the 6MWD for ID participants between the baseline and the last available measurement. Activity monitor analysis for both IC and ID groups, showed no significant change in the time spent per day recumbent or seated across all assessment time points. Neither was there a significant difference in the number of steps taken by the IC group between the baseline and the end of the study. However, participants in this group increased their daily physical activity in the second half of the study (mean change between 3 months and end of study was $983 \pm 454$ steps, $\mathrm{P}=0.023$ ). The lack of significance between baseline and completion appeared to be from fewer steps taken at the three-month assessment compared to the baseline ( $\mathrm{P}=0.262)$. Chest press muscle strength was increased at the end of study compared to baseline in the IC group $(\mathrm{P}=0.045)$. There were no significant changes in knee $(\mathrm{P}=0.324)$ or hip extension strength $(\mathrm{P}=0.057)$.

\section{Discussion}

There were several interesting findings from this pilot study. First, the baseline characteristics suggest hemodialysis patients have a poor HRQoL compared to the national average and a high prevalence of depression. Secondly, patients on hemodialysis were sedentary with significantly reduced exercise capacity and muscle strength. Thirdly, there was a much higher than expected drop-out rate from the intervention group. Interestingly, those who dropped out appeared to have worse physical and psychological functioning at baseline compared to participants who completed the study. Finally, participants who completed the study did show an improved HRQoL, but this was restricted to the physical functioning domain. This improvement in physical func- 
Table 3. Changes in Outcomes Between Baseline and Study End

\begin{tabular}{|c|c|c|}
\hline & Mean change $(95 \% \mathrm{CI})$ & Pvalue \\
\hline \multicolumn{3}{|l|}{ CDS } \\
\hline $\mathrm{IC}$ & $-7.33(-25.00-10.31)$ & 0.334 \\
\hline ID & $-2.38(-16.75-12.00)$ & 0.708 \\
\hline $\mathrm{CO}$ & $2.29(-14.56-20.13)$ & 0.751 \\
\hline \multicolumn{3}{|l|}{ EES } \\
\hline $\mathrm{IC}$ & $3.83(-38.07-45.73)$ & 0.823 \\
\hline ID & $-18.38(-64.26-27.51)$ & 0.375 \\
\hline $\mathrm{CO}$ & $17.38(-31.08-65.83)$ & 0.425 \\
\hline \multicolumn{3}{|c|}{ 6MWD (m) } \\
\hline $\mathrm{IC}$ & $98(24-172)$ & 0.018 \\
\hline ID & $-12(-103-80)$ & 0.769 \\
\hline \multicolumn{3}{|c|}{ Time spent recumbent (h) } \\
\hline $\mathrm{IC}$ & $0.15(-0.86-1.16)$ & 0.730 \\
\hline ID & $0.70(-0.29-1.70)$ & 0.123 \\
\hline \multicolumn{3}{|c|}{ Steps per day } \\
\hline $\mathrm{IC}$ & $193(-2,009-2,395)$ & 0.857 \\
\hline ID & $-1,286(-3,110-573)$ & 0.128 \\
\hline \multicolumn{3}{|c|}{ Chest press strength $(\mathrm{kg})$} \\
\hline IC & $5.21(0.15-10.27)$ & 0.045 \\
\hline ID & $3.42(-15.31-8.47)$ & 0.248 \\
\hline \multicolumn{3}{|c|}{ Knee extension strength (kg) } \\
\hline $\mathrm{IC}$ & $2.50(-3.20-8.22)$ & 0.324 \\
\hline ID & $-4.73(-9.55-0.08)$ & 0.053 \\
\hline \multicolumn{3}{|c|}{ Hip extension strength (kg) } \\
\hline $\mathrm{IC}$ & $1.33(0.05-2.70)$ & 0.057 \\
\hline ID & $-1.49(-5.30-2.31)$ & 0.300 \\
\hline
\end{tabular}

tioning matched the improved 6MWD, muscle strength, and physical activity for the participants who completed exercise training during the last 3 months of the program.

Depending on the psychometric method used, previous studies have shown that the prevalence of depression amongst hemodialysis patients ranges between $20 \%$ and $30 \%[4,7]$. Our study used the CDS, a tool validated in populations with cardiac disease to screen for depression and adjustment disorder with depressed mood $[11,18]$. Even though the CDS was not specifically designed for the ESKD population, there are similarities. Both severe cardiac disease and dialysis represent chronic disease and adjustment to dialysis dependence has been shown to be related to a real or perceived loss of health and functioning [19]. Using CDS, we found 39\% of the participants had a score that corresponded to a diagno- sis of severe depression, but only $11 \%$ of patients had been diagnosed with depression. This result confirms the high prevalence of depression in this population and suggests a possible emotional component to the observed low physical activity levels. Our study also highlights the fact that depression is under-diagnosed in the ESKD population.

The drop-out rate $(70 \%)$ was higher than the previously reported findings of between $7 \%$ and $11 \%$ in the ESKD population $[4,20]$. Most participants left the study during the initial phase when CBT was the only form of intervention. While most drop-outs were stated to be due to difficulty meeting the time commitment, a significant number (4/17) also cited a lack of perceived benefit from the psychological sessions as their primary reason for leaving the intervention $\mathrm{arm}$. Adherence to treatment is a well-documented problem 


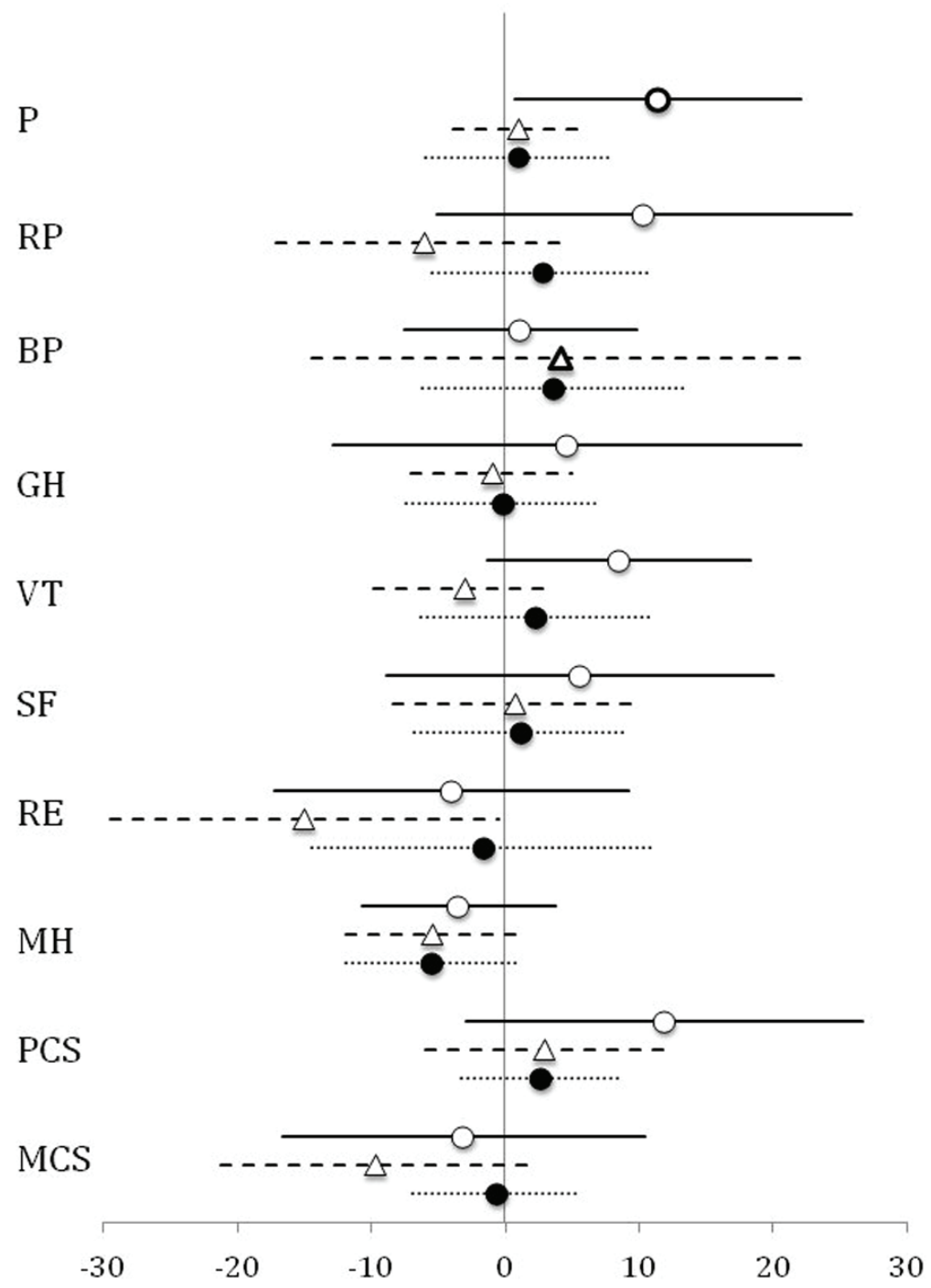

Figure 3. Mean change in SF-36 scores between baseline and the end of study. A positive value indicates an improvement at the end of study. The horizontal lines indicate the $95 \%$ confidence intervals. IC: white circle; ID: white triangle; CO: black circles.

in chronic disease populations and in mental health settings [21]. For people with diabetes or CKD, reported adherence to self-management and lifestyle change regimens is about $50 \%[22,23]$. However, psychotherapy studies indicate that early drop-out is common, with a quarter withdrawing before the first session, and half withdrawing before the fifth [23]. Possible predictors of non-adherence drawn from these studies include lower self-efficacy [24], younger age [25], lower self-rated importance of initiating psychotherapy [26], co-morbidities $[24,125]$ and provider characteristics (for example therapeutic alliance) [24]. Validation studies need to be performed to confirm these reported findings.

It is interesting that even at baseline patients who dropped out had a trend towards a lower mental composite summary score on the SF-36v2. The ID group had especially low RE scores, which was significant even after excluding drop-outs due to significant physical illness. It is possible that participants who theoretically have the most to gain are also those most likely to withdraw from the intervention. The reasons for this are unclear but may relate to lower self-efficacy, as suggested by the lower EES score. Self-efficacy is defined as an individual's personal beliefs regarding their capabilities to carry out a specific task to achieve a desired outcome [26]. Numerous studies on chronic medical illnesses have demonstrated an association between low self-efficacy and poor adherence to treatment [27].

For those who completed the interventions (IC group), significant improvements were noted primarily within the physical domains, as suggested by the improvement in SF36 physical functioning domain, an increased $6 \mathrm{MWD}$, and 
an increase in daily steps over the last 3 months of the study. While the improvement may be directly related to the physical exercise interventions, it is also possible that CBT contributed positively by addressing the participants' negative perception of their exercise capacity. In contrast to the physical parameters, we did not observe any significant improvements in markers of psychological well-being, even though there was a numerical improvement in CDS. This may be a type II error given the positive results from previous studies $[4,5,18]$.

This study had several limitations. Even as a pilot study, the sample size was unavoidably small. Findings are therefore prone to type II errors due to lack of statistical power. Selection bias may have confounded the result given the recruitment method. Patients were first asked to be in the intervention group. Hence, the control group came from a pool of patients who had already refused intervention. It is possible that such patients were physically and psychologically different from those consented, although we did not find significant differences in their baseline characteristics on testing. The high drop-out from the psychological intervention likely also affected the fidelity of the CBT program. It was designed for a group but in some cases ended up being delivered to one or two participants. Due to the high drop-out rate, we performed the primary analysis based on a protocolachieved basis and the results should be interpreted accordingly.

\section{Conclusion}

In conclusion, a combined psychological and exercise training program may benefit well-being in hemodialysis patients. Benefits in this study at least were observed only in those who completed the protocol. Further studies should aim to better understand the antecedents of depression and psychological adjustment in the hemodialysis population to ensure effective targeting of psychological support and appropriate packaging of combined physical and psychological intervention. The study concurs with the already strong evidence that hemodialysis is associated with a heavy psychological burden as reflected in high rates of depression. However, there is a need to address the optimal method of providing psychological support to a group already burdened with physical ill health, extended treatment times and medical appointments. Psychotherapy delivered online or with telephone support, shown to be effectives in other populations, may also hold promise of increased adherence for hemodialysis patients and might better demonstrate the benefits of combined intervention in these patients.

\section{Acknowledgement}

We would like to thank Amgen for an unrestricted grant which enabled the project to proceed.

\section{References}

1. Briggs N, Hurst K, McDonald S. Chapter 4: Method and Location of Dialysis, ANZDATA Registry report 2011, Australia and New Zealand and Transplant Registry. 2012.

2. Fukuhara S, Lopes AA, Bragg-Gresham JL, Kurokawa K, Mapes DL, Akizawa T, Bommer J, et al. Healthrelated quality of life among dialysis patients on three continents: the Dialysis Outcomes and Practice Patterns Study. Kidney Int. 2003;64(5):1903-1910.

3. Lovelock, H., Matthews, R. and Murphy, K. (eds). Evidence Based Psychological Interventions in the Treatment of Mental Disorders: A Literature Review (3rd Ed). Melbourne: Australian Psychological Society Pty Ltd.

4. Duarte PS, Miyazaki MC, Blay SL, Sesso R. Cognitivebehavioral group therapy is an effective treatment for major depression in hemodialysis patients. Kidney Int. 2009;76(4):414-421.

5. Kaniarz EG. Depression and anxiety: a cognitive behavioral therapy group for dialysis patients - a case study of two seven-week sessions. J Nephrol Soc Work. 1998;18:123-128.

6. Cukor D, Coplan J, Brown C, Friedman S, Newville H, Safier M, Spielman LA, et al. Anxiety disorders in adults treated by hemodialysis: a single-center study. Am J Kidney Dis. 2008;52(1):128-136.

7. Cukor D, Peterson RA, Cohen SD, Kimmel PL. Depression in end-stage renal disease hemodialysis patients. Nat Clin Pract Nephrol. 2006;2(12):678-687.

8. Lii YC, Tsay SL, Wang TJ. Group intervention to improve quality of life in haemodialysis patients. J Clin Nurs. 2007;16(11C):268-275.

9. Ouzouni S, Kouidi E, Sioulis A, Grekas D, Deligiannis A. Effects of intradialytic exercise training on healthrelated quality of life indices in haemodialysis patients. Clin Rehabil. 2009;23(1):53-63.

10. Hawthorne G. Chapter 5: Australian SF-36 V2 norms and the impact of incontinence on health status. Measuring incontinence in Australia. 2006, Commonwealth of Australia.

11. Hare DL, Davis CR. Cardiac Depression Scale: validation of a new depression scale for cardiac patients. J Psychosom Res. 1996;40(4):379-386.

12. Everett B, Salamonson Y, Davidson PM. Bandura's exercise self-efficacy scale: validation in an Australian cardiac rehabilitation setting. Int J Nurs Stud. 2009;46(6):824-829.

13. Fitts SS, Guthrie MR. Six-minute walk by people with chronic renal failure. Assessment of effort by perceived exertion. Am J Phys Med Rehabil. 1995;74(1):54-58. 
14. Godfrey A, Culhane KM, Lyons GM. Comparison of the performance of the activPAL Professional physical activity logger to a discrete accelerometer-based activity monitor. Med Eng Phys. 2007;29(8):930-934.

15. Shi WY, Stewart AG, Hare DL. Major depression in cardiac patients is accurately assessed using the cardiac depression scale. Psychother Psychosom. 2010;79(6):391392.

16. Enright PL, Sherrill DL. Reference equations for the sixminute walk in healthy adults. Am J Respir Crit Care Med. 1998;158(5 Pt 1):1384-1387.

17. Bohannon RW. Reference values for extremity muscle strength obtained by hand-held dynamometry from adults aged 20 to 79 years. Arch Phys Med Rehabil. 1997;78(1):26-32.

18. Cukor D. Use of CBT to treat depression among patients on hemodialysis. Psychiatr Serv. 2007;58(5):711-712.

19. Chan R, Brooks R, Erlich J, Chow J, Suranyi M. The effects of kidney-disease-related loss on long-term dialysis patients' depression and quality of life: positive affect as a mediator. Clin J Am Soc Nephrol. 2009;4(1):160-167.

20. Chen HY, Cheng IC, Pan YJ, Chiu YL, Hsu SP, Pai MF, Yang JY, et al. Cognitive-behavioral therapy for sleep disturbance decreases inflammatory cytokines and oxidative stress in hemodialysis patients. Kidney Int. 2011;80(4):415-422.

21. World Health Organisation. Adherence to Long-term Therapies: Evidence for Action. Non-communicable Diseases and Mental Health Adherence to long-term therapies project, Geneva: World Health Organisation, 2003.

22. Delmater, A.M. Improving Patient Adherence. Clinical Diabetes. 2006;24:75-77.

23. Kammerer J, Garry G, Hartigan M, Carter B, Erlich L. Adherence in patients on dialysis: strategies for success. Nephrol Nurs J. 2007;34(5):479-486.

24. Schweitzer RD, Head K, Dwyer JW. Psychological factors and treatment adherence behavior in patients with chronic heart failure. J Cardiovasc Nurs. 2007;22(1):7683.

25. Arnow BA, Blasey C, Manber R, Constantino MJ, Markowitz JC, Klein DN, Thase ME, et al. Dropouts versus completers among chronically depressed outpatients. J Affect Disord. 2007;97(1-3):197-202.

26. Bandura A. Human agency in social cognitive theory. Am Psychol. 1989;44(9):1175-1184.

27. O’Leary A. Self-efficacy and health. Behav Res Ther. 1985;23(4):437-451. 